Pesq. Vet. Bras. 37(12):1499-1504, dezembro 2017 DOI: $10.1590 / \mathrm{S} 0100-736 \mathrm{X} 2017001200022$

\title{
Dioctofimatose renal bilateral e disseminada em cão ${ }^{1}$
}

\author{
Carolina F. Sapin ${ }^{2 *}$, Luisa C. Silva-Mariano ${ }^{2}$, Luisa Grecco-Corrêa ${ }^{2}$, Josaine \\ C.S. Rappeti ${ }^{3}$, Luana H. Durante ${ }^{3}$, Soliane C. Perera ${ }^{3}$, Marlete B. Cleff ${ }^{3}$ \\ e Fabiane B. Grecco ${ }^{2}$
}

\begin{abstract}
Sapin C.F., Silva-Mariano L.C., Grecco-Corrêa L., Rappeti J.C.S., Durante L.H., Perera S.C., Cleff M.B. \& Grecco F.B. 2017. [Bilateral and disseminated renal dioctophimatosis in dog.] Dioctofimatose renal bilateral e disseminada em cão. Pesquisa Veterinária Brasileira 37(12):1499-1504. Departamento de Patologia Animal, Universidade Federal de Pelotas, Av. Eliseu Maciel s/n, Prédio 1, Campus Universitário Capão do Leão, Pelotas, RS 96010-900, Brazil. E-mail: carolinasapin@yahoo.com.br

This paper describes a bilateral and disseminated renal dioctophymiasis in a canine in the municipality of Pelotas, southern region of Rio Grande do Sul, in Brazil. The two-yearold female dog with no defined race was semi-domiciled and was submitted to ultrasound examination presenting alterations suggestive of parasitism by Dioctophyme renale. The patient died before being led for nephrectomy and removal of the parasites. It was referred for necropsy at the Regional Diagnostic Laboratory of the UFPel Veterinary School. Macroscopically, at the cut of the right kidney, atrophy of the parenchyma with cystic formations and serosanguinous exudate with four female specimens of $D$. renale were observed. The renal capsule was $0.4 \mathrm{~cm}$ thick and had irregular, hard, multifocal regions measuring up to $2 \mathrm{~cm}$ in diameter. In the pelvis of the left kidney, there was a $22 \mathrm{~cm}$ long male parasite. The bladder had darkened and pasty contents. Also, free in the abdominal cavity, near to the right kidney there was another male parasite of $30 \mathrm{~cm}$. In the liver there was a cicatricial region in the lobe in contact with the parasite. In the thorax, a male parasite of $D$. renale measuring $20 \mathrm{~cm}$, was identified free in the cavity and forming an impression under the pericardial fat. Histologically, in addition to the characteristic lesions of the disease, bone metaplasia was observed in the right renal capsule.
\end{abstract}

INDEX TERMS: Dioctophyme renale, dioctophimatosis, kidney, bone metaplasia, bilateral renal, dog.

RESUMO.- Este trabalho descreve dioctofimatose renal bilateral e disseminada em canino no município de Pelotas, na região sul do Rio Grande do Sul. Uma cadela sem raça definida, de dois anos de idade e semi-domiciliada foi submetida a exame ultrassonográfico observando-se alterações sugestivas de parasitismo por Dioctophyme renale. 0 animal veio à morte antes de ser conduzido para nefrectomia e remoção dos parasitas. Foi encaminhado para necropsia no Laboratório Regional de Diagnóstico da Faculdade de Veteri-

\footnotetext{
${ }^{1}$ Recebido em 27 de dezembro de 2016.

Aceito para publicação em 20 de abril de 2017.

${ }^{2}$ Departamento de Patologia Animal, Universidade Federal de Pelotas (UFPel), Av. Eliseu Maciel s/n, Campus Universitário, Capão do Leão, Pelotas, RS 96010-900, Brasil.*Autor para correspondência: carolinasapin@ yahoo.com.br

${ }^{3}$ Hospital de Clinicas Veterinária, UFPel, Av. Eliseu Maciel s/n, Campus Universitário, Capão do Leão, Pelotas, RS 96010-900, Brasil.
}

nária da UFPel. Macroscopicamente, ao corte do rim direito observou-se atrofia do parênquima com formações císticas e exsudato serosanguinolento com quatro exemplares fêmeas de $D$. renale. A cápsula renal media $0,4 \mathrm{~cm}$ de espessura, e apresentava placas multifocais esbranquiçadas, irregulares e de consistência dura, medindo até $2 \mathrm{~cm}$ de diâmetro. $\mathrm{Na}$ pelve do rim esquerdo, havia um parasita macho de $22 \mathrm{~cm}$. A bexiga possuía conteúdo escurecido e pastoso. Ainda, livre na cavidade abdominal, próximo ao rim direito havia outro parasita macho de $30 \mathrm{~cm}$. No fígado havia região cicatricial no lobo em contato com o parasita. No tórax identificou-se livre na cavidade e formando impressão sob a gordura pericárdica, um parasita macho de D. renale, medindo $20 \mathrm{~cm}$. Histologicamente, além de lesões características da enfermidade, observou-se metaplasia óssea na capsula renal direita.

TERMOS DE INDEXAÇÃO: Dioctophyme renale, dioctofimatose, rim, metaplasia óssea, renal bilateral, canino. 


\section{INTRODUÇÃO}

Dioctophyme renale é um nematóide de distribuição mundial, conhecido como o verme gigante do rim (Pedrassani \& Nascimento 2015). Os hospedeiros definitivos de D. renale são mustelídeos, carnívoros domésticos e silvestres, embora os cães sejam considerados de pouca importância para o ciclo, estes podem ser hospedeiros definitivos, terminais ou anormais (Kommers et al. 1999), podendo também acometer o homem, caracterizando-se como zoonose (Pedrassani \& Nascimento 2015). O ciclo evolutivo deste parasita ocorre pela ingestão de ovos pelo hospedeiro intermediário, o anelídeo oligoqueta aquático conhecido como Lumbriculus variegatus. Após, a larva no interior do ovo se desenvolve até o estágio infectante (L3), podendo causar a parasitose no hospedeiro definitivo quando este se alimenta do anelídeo ou dos hospedeiros intermediários e paratênicos infectados, como peixes de água doce, sapos e rãs (Kommers et al. 1999, Nakagawa et al. 2007, Verocai et al. 2009, Pedrassani \& Nascimento 2015).

D. renale acomete o rim, principalmente o direito, penetra na cápsula renal e invade o parênquima, destruindo completamente o órgão. 0 parasita pode ser encontrado no peritônio, glândula mamária, bolsa escrotal, tecido subcutâneo, útero, ovário, cavidade torácica, pericárdio, linfonodo mesentérico e no espaço intra-dural (Kommers et al. 1999, Pedrassani \& Nascimento 2015, Bach et al. 2016). Os parasitas adultos se reproduzem normalmente no rim direito e as fêmeas liberam os ovos através da urina (Pedrassani \& Nascimento 2015).

Os sinais clínicos costumam ser inespecíficos ou até mesmo ausentes, principalmente em parasitismo renal unilateral devido à compensação realizada pelo rim contralateral (Kommers et al. 1999). Quando observados, os sinais clínicos consistem em disúria, hematúria e dor lombar (Pedrassani \& Nascimento 2015, Silveira et al. 2015). 0 diagnóstico é baseado no exame ultrassonográfico e na detecção de ovos na urina, ou através do achado incidental de necropsia (Pedrassani \& Nascimento 2015). 0 tratamento é caracterizado pela nefrectomia e remoção cirúrgica dos parasitas (Sousa et al. 2011).

Regiões com grande potencial hídrico possuem maiores chances de ocorrência da dioctofimatose pelo fácil acesso dos animais aos hospedeiros paratênicos ou intermediários do nematódeo (Figueiredo et al. 2013). 0 objetivo deste trabalho é descrever a ocorrência de dioctofimatose renal bilateral e disseminada em cães em Pelotas, na região sul do Rio Grande do Sul.

\section{MATERIAL E MÉTODOS}

Os dados epidemiológicos, sinais clínicos e evolução da dioctofimatose em uma cadela, foram obtidos junto ao clínico responsável. A necropsia foi realizada no Laboratório Regional de Diagnóstico e foram registradas as alterações macroscópicas. Os fragmentos de órgãos foram fixados em formalina tamponada $10 \%$, processadas rotineiramente, cortados em secções de $3 \mu \mathrm{e}$ coradas com a técnica de hematoxilina e eosina (HE) para análise histopatológica.

\section{RESULTADOS}

Foi atendido no ambulatório veterinário do Ceval do Hospital de Clínicas Veterinárias da Universidade Federal de Pelotas (UFPel), cadela, sem raça definida, de dois anos de idade, semi-domiciliada, com suspeita de corpo estranho intestinal. Apresentava desconforto abdominal, fezes sanguinolentas e anúria há 24 horas, o que impossibilitou o exame de urinálise. No hemograma havia leucocitose com desvio a esquerda e elevação dos compostos nitrogenados (ureia), a valores que excediam os padrões aferidos pelo aparelho utilizado para análise. 0 animal foi encaminhado para o exame ultrassonográfico observando-se alteração do formato anatômico do rim direito, com dimensões aumentadas e contorno irregular. Evidenciou-se a presença de estruturas tubulares apresentando finas paredes hiperecogênicas, ocupando todo parênquima do órgão sugerindo o parasitismo por Dioctophyme renale. 0 rim esquerdo apresentava formato anatômico preservado, dimensões aumentadas e contorno irregular; a relação córtico-medular apresentava-se alterada, com a cortical espessada, hiperecogênica, heterogênea e perda de definição córtico-medular. A pelve renal estava preservada. Observou-se ainda estrutura tubular apresentando finas paredes hiperecogênicas, sugerindo também o parasitismo por $D$. renale. Não foram identificadas outras alterações nos demais órgãos da cavidade abdominal. Foi estabelecida terapia de suporte com antibióticos e fluidoterapia. 0 animal veio à morte antes de ser conduzido para nefrectomia e remoção dos parasitas. Foi encaminhado para necropsia no Laboratório Regional de Diagnóstico da Faculdade de Veterinária da UFPel.

$\mathrm{Na}$ abertura da cavidade abdominal observou-se que o omento estava enegrecido. Macroscopicamente, os rins eram esbranquiçados e amolecidos. Ao corte do rim direito observou-se ausência do parênquima, o qual estava cístico e com abundante exsudato serosanguinolento. Havia quatro exemplares fêmeas de D. renale, que mediam $8 \mathrm{~cm}$, $34 \mathrm{~cm}, 37,5 \mathrm{~cm}$ e $67 \mathrm{~cm}$, respectivamente (Fig.1). A cápsula

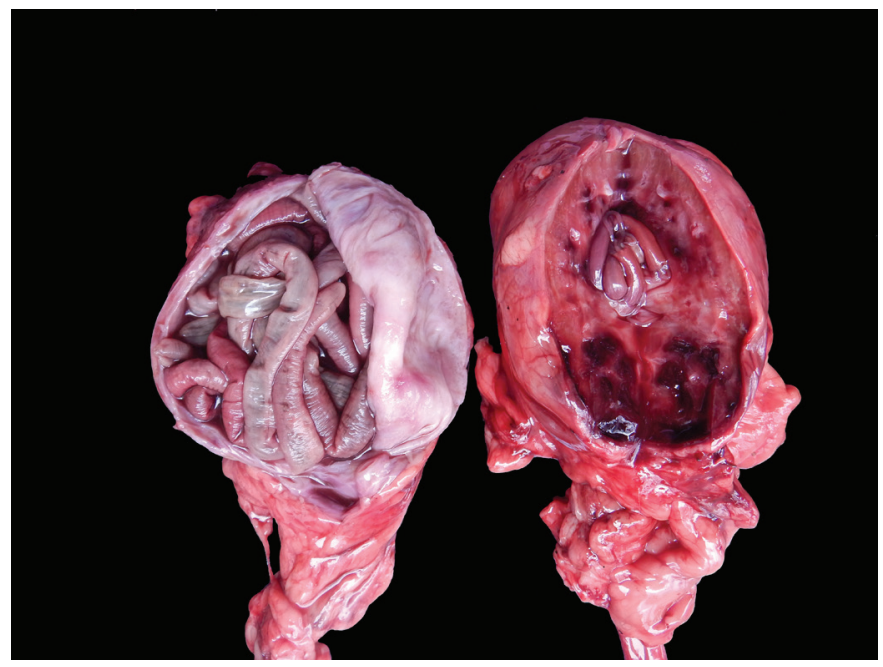

Fig.1. Parasitismo renal bilateral por Dioctophyme renale em cão. 0 rim direito apresenta atrofia acentuada do parênquima. No rim esquerdo observa-se comprometimento da pelve renal. 


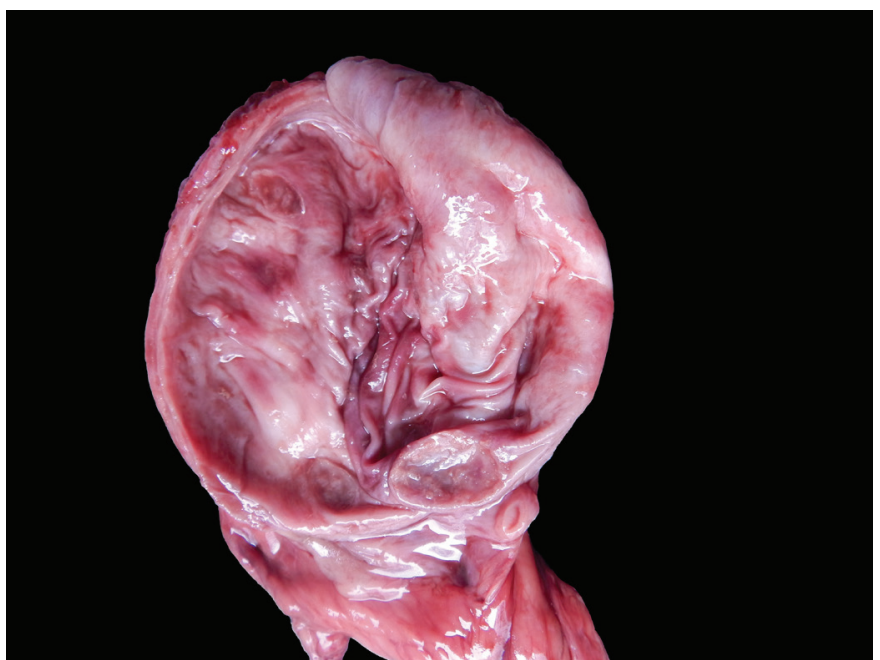

Fig.2. Rim direito de cão parasitado por Dioctophyme renale. Notam-se placas multifocais esbranquiçadas, irregulares e de consistência dura.

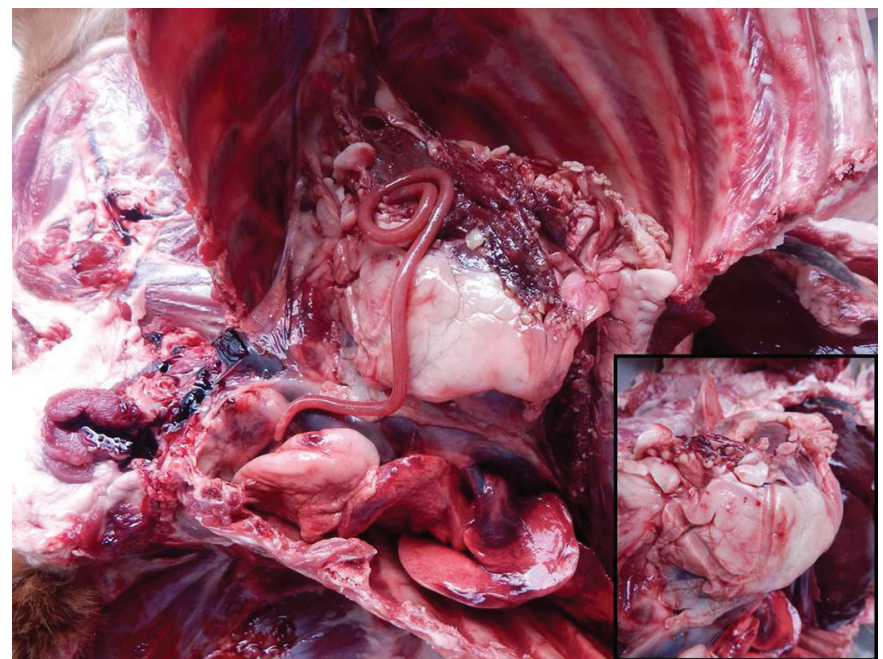

Fig.3. Parasitismo torácico por Dioctophyme renale em cão. 0 parasita está alojado sobre a gordura pericárdica e ao ser removido é possível observar a impressão deste.

renal media $0,4 \mathrm{~cm}$ de espessura, e apresentava placas multifocais esbranquiçadas, irregulares e de consistência dura, variando de 0,5 a $2 \mathrm{~cm}$ de diâmetro (Fig.2). Na pelve do rim esquerdo, havia um parasita macho de $22 \mathrm{~cm}$. Notavam-se áreas avermelhadas e amolecidas distribuídas pelo parênquima. Ainda observou-se aderência e espessamento da cápsula renal esquerda com hemorragia subcapsular. A bexiga possuía conteúdo escurecido e pastoso. No baço havia áreas de cicatriz. Ainda, livre na cavidade abdominal, próximo ao rim direito havia outro parasita macho de $30 \mathrm{~cm}$. No fígado havia região cicatricial no lobo em contato com o parasita.

No tórax identificou-se livre na cavidade e formando impressão sob a gordura pericárdica, um parasita macho de $D$. renale, medindo $20 \mathrm{~cm}$ (Fig.3). No parênquima pulmonar direito, havia dois nódulos vermelho-enegrecidos. 0 diafragma apresentava múltiplos nódulos esbranquiçados aderidos e avermelhados, por vezes de aspecto fibrinoso.
Na análise histopatológica do rim direito havia perda do parênquima renal, com redução do número de glomérulos e túbulos renais. Observou-se nefrite caracterizada por infiltrado de células mononucleares e presença de cilindros hialinos. Havia fibroplasia intensa e difusa em resposta a destruição do parênquima. Ovos de Dioctophyme renale, caracterizados por serem elípticos, refringentes, acastanhados, bioperculados e com depressões exceto nos polos foram observados. Metaplasia óssea multifocal foi identificada em áreas da cápsula renal (Fig.4). 0 rim esquerdo apresentava proliferação moderada de tecido conjuntivo fibroso na pelve renal e deposição de ovos de Dioctophyme renale.

$\mathrm{Na}$ luz e parede da bexiga, observou-se acentuada presença de ovos do parasita (Fig.5), os quais também foram identificados na luz dos ureteres (Fig.6). Ovos foram encontrados também no parênquima pulmonar, sem resposta tecidual. Ainda observou-se peritonite piogranulomatosa.

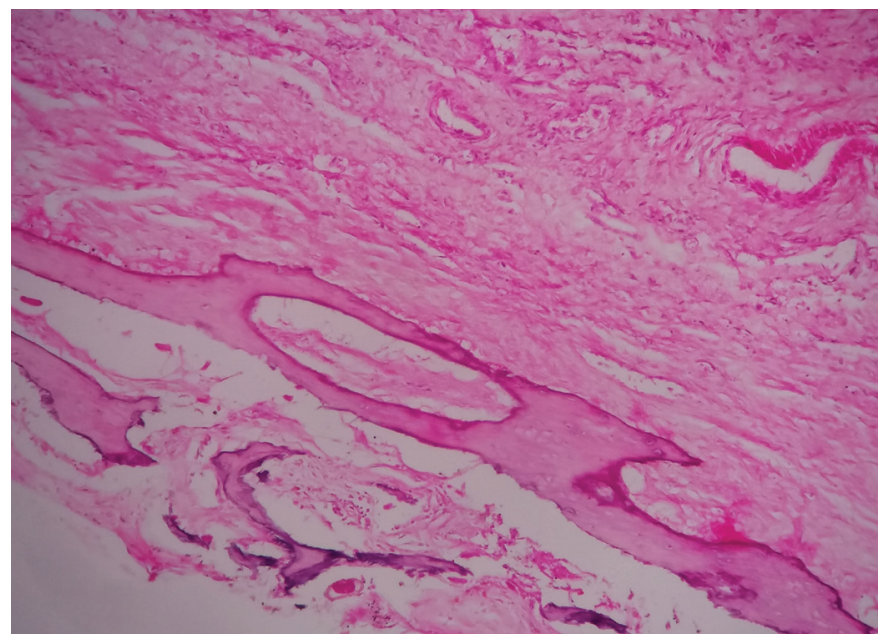

Fig.4. Metaplasia óssea do parênquima do rim direito de cão parasitado por Dioctophyme renale. HE, obj.10x.

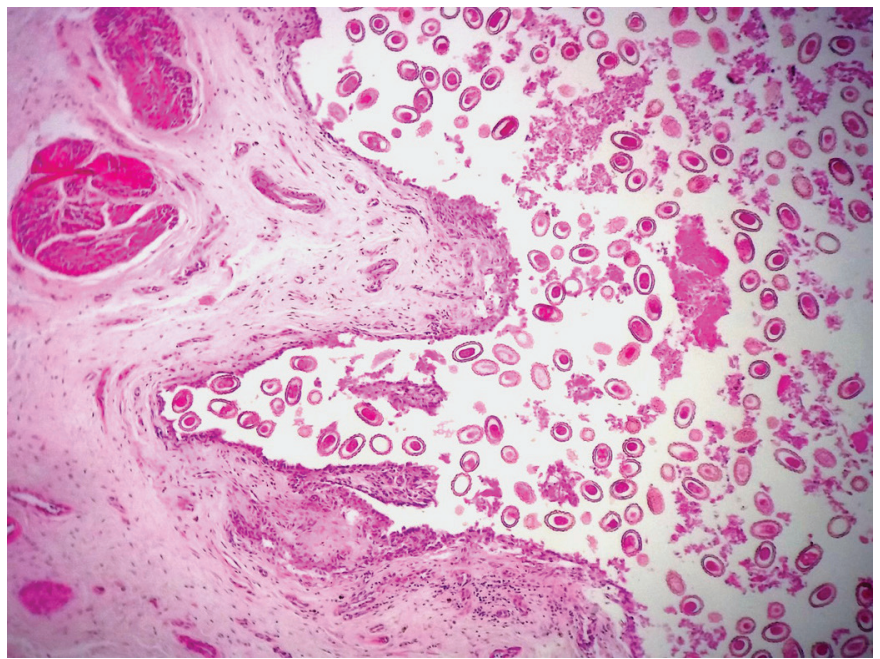

Fig.5. Bexiga de cão parasitado por Dioctophyme renale. A luz do órgão exibe numerosos ovos elípticos e bioperculados do parasita. HE, obj.5x. 


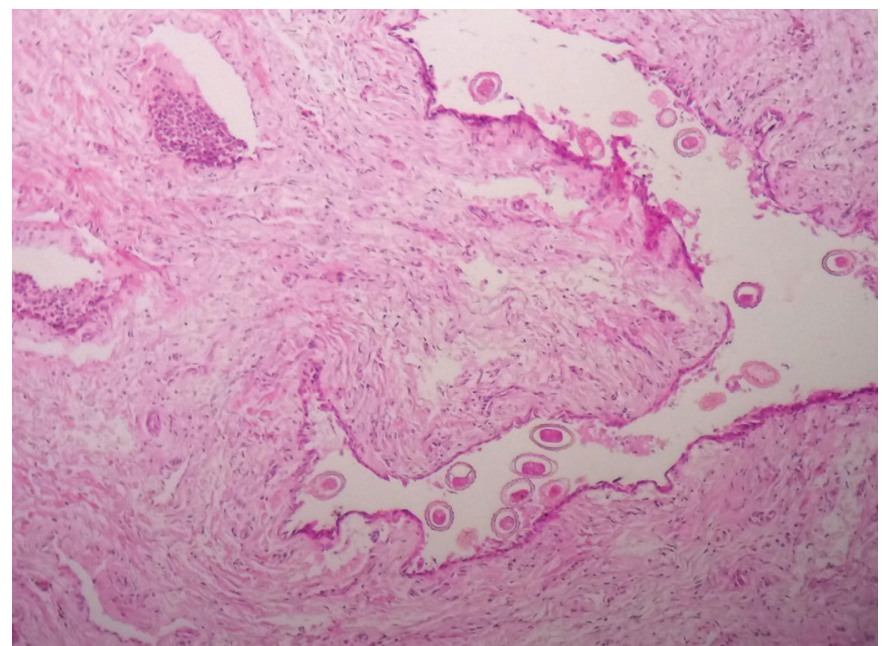

Fig.6. Ureter esquerdo de cão parasitado por Dioctophyme renale. Observa-se na luz do órgão ovos elípticos e bioperculados do parasita. HE, obj.10x.

\section{DISCUSSÃO}

0 rim direito é o local de maior frequência para o parasitismo por Dioctophyme renal. Outras localizações menos frequentes do parasita adulto são o rim esquerdo, bexiga, uretra, bolsa escrotal, glândula mamária, tecido subcutâneo inguinal, útero, ovário, linfonodo mesentérico e pericárdio (Kommers et al. 1999, Monteiro et al. 2002, Zabott et al. 2012, Pedrassani \& Nascimento 2015). 0 parasitismo bilateral renal é raro.

0 local onde a larva penetra no trato gastro-intestinal do hospedeiro definitivo determina a localização do parasita adulto. Ao penetrar na curvatura menor do estômago, as larvas evoluem para a forma adulta entre os lobos hepáticos, o que possibilita que o parasita se encontre de forma livre nas cavidades corpóreas. Quando penetram na curvatura maior do estômago, tendem a se localizar no rim esquerdo; já quando na parede duodenal, desenvolvem-se no rim direito (Kommers et al. 1999, Zabott et al. 2012). Neste trabalho, observou-se presença dos parasitas em ambos os rins e nas cavidades torácica e abdominal, caracterizando uma forma disseminada do parasitismo por D. renale. Sugere-se que o parasitismo do rim esquerdo neste relato possa ter ocorrido via ascendente, uma vez que o parasita encontrado no rim esquerdo era um exemplar macho e o ureter esquerdo encontrava-se repletos de ovos do parasita.

0 parasitismo na cavidade torácica em cães tem sido descrito associado à consequente lesão de diafragma e pneumotórax (Pesenti et al. 2007). Neste estudo foram observadas lesões diafragmáticas, porém o animal não manifestava sinais clínicos de pneumotórax o que provavelmente esteja relacionado ao tempo de evolução.

A identificação dos exemplares foi baseada nas características morfológicas tais como coloração, formato e tamanho. Os parasitas adultos têm morfologia cilíndrica e coloração corpórea avermelhada devido à prática de hematofagia (Pedrassani \& Nascimento 2015). Os parasitas machos podem atingir até $45 \mathrm{~cm}$ comprimento e as fêmeas até $100 \mathrm{~cm}$, e $0,4 \mathrm{~cm}$ a $1,2 \mathrm{~cm}$ de largura (Sousa et al. 2011). Quanto ao sexo os machos apresentam bolsas copuladoras na extremidade posterior em forma de sino e um espículo com aproximadamente $0,6 \mathrm{~cm}$ de comprimento. Já as fêmeas possuem a extremidade posterior romba, ânus terminal, um ovário e vulva, distando entre $5 \mathrm{a} 7 \mathrm{~cm}$ da extremidade anterior (Barriga 1982). No presente relato foram encontrados quatro parasitas fêmeas e três machos.

Os animais afetados pelo parasitismo podem ser assintomáticos ou apresentarem sinais clínicos inespecíficos que variam conforme a localização, o grau de desenvolvimento do parasita e a reação dos tecidos lesados, bem como a espécie animal parasitada (Dacorso-Filho et al. 1954). Alguns animais podem exibir sinais de peritonite ou uremia devido à insuficiência renal estabelecida. Hematúria, disúria, anúria, gastrite e enterite ulcerativa e hemorrágica são alguns dos sinais clínicos associados à insuficiência renal estabelecida. Ainda podem ser observados anemia e emagrecimento progressivo (Newman 2013). Quando apenas um rim é acometido, a função renal é preservada pelo rim contralateral (Kommers et al. 1999). Os sinais clínicos descritos no presente relato eram característicos de síndrome uremica em consequência de insuficiência renal crônica devido ao comprometimento bilateral dos rins.

A confirmação do diagnóstico é realizada através da necropsia ou por exames complementares como a ultrassonografia e urinálise (Pedrassani \& Nascimento 2015). Desta forma, a ultrassonografia da região abdominal é fundamental, uma vez que permite identificar alterações e a presença de estruturas compatíveis com $D$. renale no trato urinário e nos outros órgãos e o pronto estabelecimento do tratamento preconizado (Pedrassani \& Nascimento 2015). A principal característica macroscópica da parasitose é a destruição das camadas medular e cortical renais pela ação das enzimas esofágicas do parasita, reduzindo o órgão a uma capsula fibrosa. Porem essa destruição também esta associada à hidronefrose causada pelo alojamento do exemplar e consequente obstrução pélvica (Nakagawa et al. 2007). 0 rim direito apresentava características mais evidentes do parasitismo por D. renale, com destruição das camadas medular e cortical estando reduzido a uma capsula fibrosa. Ainda era possível observar áreas esbranquiçadas, irregulares e de consistência dura, devido a constante agressão da cápsula fibrosa pelo parasita. Apesar da agressividade do parasita, o rim esquerdo apresentava apenas lesão na pelve, provavelmente devido ao tempo de evolução, uma vez que o cão veio à morte antes da progressão do processo, neste rim.

Alterações histopatológicas como necrose, reação inflamatória granulomatosa ou mononuclear, atrofia e fibrose do parênquima renal, caracterizam o parasitismo pelo nematoide em cães (Kommers et al. 1999). A metaplasia óssea observada no presente estudo é descrita na parede dos rins de furões e visions (Mace 1975) e raramente em cães (Sapin et al. 2016, 2017), sendo que esta ocorre pela constante agressão da parede renal pelas enzimas proteolíticas e lipolíticas produzidas pelas glândulas esofágicas de D. renale.

Diversos autores indicam o tratamento cirúrgico com nefrectomia, quando apenas um rim é acometido, e nefrotomia quando ambos os rins são acometidos. Ainda pode ser realizada a remoção cirúrgica do parasita (Kommers et al. 1999, Sousa et al. 2011, Ferreira et al. 2010). 
Em humanos, a dioctofimatose está associada à ingestão de peixes ou rãs mal cozidos. Embora a maioria dos peixes destinados à alimentação humana seja eviscerada, larvas L3 do parasita podem ser encontradas na musculatura dos peixes, evidenciando um potencial risco para consumidores e sendo dessa forma uma parasitose de importância em saúde publica (Pedrassani \& Nascimento 2015). Na literatura há diversos relatos da parasitose em humanos (Urano et al. 2001, Ignjatovic et al. 2003), inclusive da parasitose renal bilateral (Li et al. 2010).

A grande frequência da doença em cães errantes ou semi-domiciliados sugere que o parasitismo esteja relacionado aos hábitos alimentares pouco seletivos desses animais (Silveira et al. 2015). 0 cão deste relato era semi-domiciliado. Regiões geográficas com grande potencial hídrico parecem possuir maior predisposição para casos de dioctofimatose (Pereira et al. 2006). A localização geográfica da cidade de Pelotas que é banhada pela laguna dos patos e diversos rios fazem com que a área seja propicia para o ciclo de Dioctophyme renale uma vez que propicia a alimentação com hospedeiros intermediários e paratênicos do parasita, como sapos, rãs e peixes de água doce. 0 cão do presente relato vivia em uma região alagadiça da cidade de Pelotas, com condições sanitárias precárias, o que possivelmente oportunizou a contaminação.

Há estudos que revelam o parasitismo por $D$. renale em ratazanas (Rattus norvegicus) (Tokiwa et al. 2011). Roedores domésticos possuem regime alimentar amplo, vivem e se reproduzem em locais próximos a água, como esgotos e áreas alagadiças, encontradas em centros habitacionais e de condições sanitárias precárias. 0 acesso à água favorece a infecção desses roedores pelo parasita. Desta forma é possível questionar outras fontes de infecção para cães errantes, uma vez que estes roedores fazem parte dos hábitos alimentares destes animais (Kommers et al. 1999).

0 parasita já foi encontrado também em diversas espécies de peixes de agua doce como Pseudoscaphirynchus kaufmanni, Ictalurus nebulosus, Ictalurus melas, Micropterus salmoides, Esox lucius, Leuciscus idus, Aspius aspius, Rutilus rutilus, Chalcalburnus chalcoides, dentre outros (Mace \& Anderson 1975, Measures \& Anderson 1985, Measures 2001). Como dito anteriormente, a cidade é banhada por aguas doce e assim sugere-se o estudo dos peixes encontrados na costa de Pelotas, destacando-se Rhamdia quelen, Astianax ssp., Cyprinus carpio e Hoplias malabaricus.

0 desenvolvimento dos ovos é necessário para a continuidade do ciclo biológico de D. renale (Freitas 1980). Os ovos de $D$. renale são dependentes da temperatura e apresentam características morfologias que podem ser importantes para o ciclo de vida do parasita, como casca espessa e com depressões. Estas depressões podem fornecer pontos para adesão de vegetação e biofilme, tornado os ovos mais atrativos para os hospedeiros intermediários (Pedrassani \& Nascimento 2015). A temperatura ideal para o embrionamento dos ovos está entre 25 e $30^{\circ} \mathrm{C}$. Após 30 dias a esta temperatura são observadas larvas de primeiro estádio. Quando expostos a temperaturas baixas, o embrionamento pode ser até sete meses (Freitas 1980). As condições climáticas da região de Pelotas, com temperaturas médias anuais em torno de $20^{\circ} \mathrm{C}$, parecem propícias ao desenvolvimento dos ovos e consequente maior ocorrência da doença. As larvas de primeiro estádio de $D$. renale desenvolvem-se em água quando incubadas em temperatura de 14 a $30^{\circ} \mathrm{C}$, com variação da taxa de desenvolvimento conforme a temperatura (Pedrassani \& Nascimento 2015).

\section{CONCLUSÃO}

A região sul do Rio Grande do Sul apresenta condições favoráveis para a ocorrência de Dioctophyme renale e os cães podem apresentar a forma disseminada do parasitismo que pode ocasionar a morte. 0 exame ultrassonográfico é um grande aliado no diagnóstico precoce do parasitismo por D. renale, evitando a disseminação do parasita.

\section{REFERÊNCIAS}

Bach F.S., Klaumann P.R. \& Montiani-Ferreira F. 2016. Paraparesis secondary to erratic migration of Dioctophyma renale in a dog. Ciência Rural 46(5):885-888.

Barriga 0.0. 1982. Dioctophymosis, p.83-92. In: Schultz M.G. (Ed.), CRC Handbook Series in Zoonoses. CRC, Florida.

Dacorso-Filho P., Langenegger J. \& Döbereiner J. 1954. Sobre a infestação e lesões anatomo patológicas produzidas por Dioctophyme renale (Goeze, 1782) em cães. Revta Vet. 8(2):35-54.

Ferreira V.L., Medeiros F.P., July J.R. \& Raso T.F. 2010. Dioctophyma renale in dog: clinical diagnosis and surgical treatament. Vet. Parasitol. 168:151155.

Figueiredo M.A.P., Silva D.F., Manrique W.G. \& Sousa A.A.R. 2013. Ciclo errático de Dioctphyme renale: relato de dois casos. Orinoquia 17(1).

Freitas M.G. 1980. Helmintologia Veterinária. 4aa ed. Rabelo, Belo Horizonte, p.267-270.

Ignjatovic I., Stojkovic I., Kutlesic C. \& Tasic S. 2003. Infestation of the human kidney with Dioctophyma renale. Urologia Internationalis, Suíça, 70(1):70-73.

Kommers G.D., Ilha M.R.S. \& Barros C.S.L. 1999. Dioctofimose em cães: 16 casos. Ciência Rural 29(3):517-522.

Li G., Liu C., Li F., Zhou M., Liu X. \& Niu Y. 2010. Fatal bilateral dioctophymatosis. J. Parasitol. 96(6):1152-1154.

Mace T.F. \& Anderson R.C. 1975. Development of the giant kidney worm, Dioctophyma renale (Goeze, 1782) (Nematoda: Dioctophymatoidea). Can. J. Zool. 53:1552-1568

Mace T.F.A. 1975. Studies on the biology of the giant kidney worm, Dioctophyma renale (Goeze,1782) (Nematoda-Dioctophymoidea). Dissertation Abstracts International 36(1):126-127.

Measures L.N. \& Anderson R.C. 1985. Centrarchid fish as paratenic hosts of the giant kidney worm, Dioctophyma renale (Goeze, 1782), in Ontario, Canada. J. Wildl. Dis. 21(1):11-19.

Measures L.N. 2001. Dioctophymatosis, p.357-364. In: Samuel W.M., Pybus M.J. \& Kocan A.A. (Eds), Parasitic Diseases of Wild Mammals. 2nd ed. Iowa State University Press, Ames.

Monteiro S.G., Sallis E.S.V. \& Stainki D.R. 2002. Infecção natural por trinta e quatro helmintos da espécie Dioctophyma renale (Goeze, 1782) em um cão. Revta FZVA, Uruguaiana, 9(1):95-99.

Nakagawa T.L.D.R., Bracarense A.P.F.R.L., Reis A.C.F., Yamamura M.H. \&Headley S.A. 2007. Giant kidney worm (Dioctophyma renale) infections in dogs from Northern Paraná, Brazil. Vet. Parasitol. 145:366-370.

Newman S.J. 2013. 0 sistema urinário, p.592-661. In: Zachary J.F. \& Mcgavin M.D. (Eds), Bases da Patologia Veterinária. Elsevier, São Paulo.

Pedrassani D. \& Nascimento A.A. 2015. Verme gigante renal. Revta Port. Ciênc. Vet. 110(593/594):30-37.

Pereira B.J., Girardelli G.L., Trivilin L.O., Lima V.R., Nunes L.C. \& Martins I.V.F. 2006. Ocorrência de Dioctofimose em cães do município de Cacho- 
eiro do Itapemirim, Espírito Santo, Brasil, no período de maio a dezembro de 2004. Revta Bras. Parasitol. Vet. 15(3):123-125.

Pesenti T.C., Krüger C., Mascarenhas C.S. \& Müller G. 2007. Ocorrência de Dioctophyma renale (Goeze, 1782) em Galictis cuja no Rio Grande do Sul. XVI Congresso de Iniciação Científica UFPel, Pelotas.

Sapin C.F., Silva-Mariano L.C., Bassi J.N. \& Grecco F.B. 2016. Anatomo-pathological and epidemiological analysis of urinary tract lesions in dogs. Ciência Rural 46(8):1443-1449.

Sapin C.F., Silva-Mariano L.C., Piovesan A.D., Fernandes C.G., Rappeti J.C.S., Braga F.V.A., Cavalcante G.A., Rosenthal B.M. \& Grecco F.B. 2017. Estudo anatomopatológico de rins parasitados por Dioctophyme renale em cães. Acta Scient. Vet. 47:1-7.

Silveira C.S., Diefenbach A., Mistieri M.L., Machado R.L. \& Anjos B.L. 2015. Dioctophyma renale em 28 cães: aspectos clinicopatológicos e ultrassonográficos. Pesq. Vet. Bras. 35(11):899-905.
Sousa A.A.R., Sousa A.A.S., Coelho M.C.O.C., Quessada A.M., Freitas M. V.M. \& Moraes R.F.N. 2011. Dioctofimose em cães. Acta Scient. Vet. 39(3):985.

Tokiwa T., Harunari T., Tanikawa T., Akao N. \& Ohta N. 2011. Dioctophyme renale (Nematoda: Dioctophymatoidea) in the abdominal cavity of Rattus norvegicus in Japan. Parasitol. Int. 60(3):324-326.

Urano Z., Hasegawa H., Katsumata T., Toriyama K. \& Aoki Y. 2001. Dioctophymatid nematode larva found from human skin with creeping eruption. J. Parasitol., Lawrence, 87(2):462-465.

Verocai G.G., Measures L.N., Azevedo F.D., Correia T.R., Fernandes J.I. \& Scott F.B. 2009. Dioctophyma renale (Goeze, 1782) in the abdominal cavity of a domestic cat from Brazil. Vet. Parasitol. 161:342-344.

Zabott M.V., Pinto S.B., Viott A.M., Tostes R.A., Bittencourt L.H.F.B., Konell A.L. \& Gruchouskei L. 2012. Ocorrência de Dioctophyma renale em Galictis cuja. Pesq. Vet. Bras. 32(8):786-788. 